\title{
Paresthesia and sensory deficits on the unilateral leg arising from an unrecognized intramedullary tumor after spinal anesthesia
}

\author{
Dae Geun Jeon, Bong Jin Kang, and Soo Mi Jeon \\ Department of Anesthesiology and Pain Medicine, College of Medicine, Dankook University, Cheonan, Korea
}

We report a case of persistent sensory deficits and paresthesia arising from intramedullary cavernous hemangioma after spinal anesthesia, suggesting that practitioners should carefully examine any neurologic abnormalities related to peri-spinal masses.

A 36-year-old male underwent an operation for a Morton's neuroma on his left foot. He was active in his daily life, and did not have back pain or any other neurologic signs. Spinal anesthesia was performed at the L3-4 interspace using a 26-gauge spinal needle with a midline approach in the left lateral position with the limb to be operated on in the dependent position. Hyperbaric bupivacaine $11 \mathrm{mg}$ was injected after a free flow of cerebrospinal fluid was observed. He was turned to the supine position, and the level of sensory loss was determined to be $\mathrm{T} 12$ by pinprick. The duration of surgery was 40 minutes, and the vital signs and peripheral oxygen saturation were stable. Post-operatively, he was transferred to the post-anesthesia care unit, and 30 minutes later, the sensory level was determined to be below T12. Therefore, he was transferred to the ward.

Five hours after spinal anesthesia, sensory function returned to normal in the left leg (operated side), but decreased sensory function and paresthesia persisted in the right leg, the right half of scrotum and the right lower abdomen. He also complained of a burning sensation, which was the most bothersome sign, on the right abdomen. Regarded as transient symptoms after spinal anesthesia, he was discharged on the third day postoperatively. Afterwards, the burning sensation on the right lower abdomen was aggravated gradually, sometimes disturbing his sleep, and the symptoms on the right leg continued without any improvements.

Even after one month's follow-up, there was no significant improvement. He was administered gabapentin $600 \mathrm{mg}$ and meloxicam $7.5 \mathrm{mg}$ a day. Observing no improvement after three weeks, pregabalin, tramadol, amitryptilline, vitamin B complex, and capsacin cream were administered, to no effect. Dye enhanced MR imaging of the lumbar spine was performed because infection or chemical meningitis was suspected. However there were no specific signs, such as a space-occupying lesion or unusual fluid collection. Sensory and motor nerve conduction studies also showed normal values. A somatosensory evoked potential study showed an increased latency to the right lateral femoral cutaneous nerve stimulation.

MR imaging showed a $1 \times 1 \times 2.3 \mathrm{~cm}$ mass located on the postero-left lateral side of spinal cord at the level of T4, and cavernous hemangioma was suspected (Fig. 1). The mass in the thoracic spinal cord was removed 4 months after spinal anesthesia. The histologic features of the mass were consistent with a cavernous hemangioma. Although the surgery was successful, the decreased sensory function has remained without any improvements through one year of follow up.

In the beginning, we considered that the cause of the patient's symptoms was neuritis or a complex regional pain syndrome. Therefore, gabapentin, pregabalin, and a non-steroidal antiinflammatory agent were administered, which were not

Corresponding author: Bong Jin Kang, M.D., Ph.D., Department of Anesthesiology and Pain Medicine, College of Medicine, Dankook University, 16-5, Anseo-dong, Cheonan 330-715, Korea. Tel: 82-41-550-6828, Fax: 82-41-550-9330, E-mail: anebjkang@dankook.ac.kr

(c) This is an open-access article distributed under the terms of the Creative Commons Attribution Non-Commercial License (http:// creativecommons.org/licenses/by-nc/3.0/), which permits unrestricted non-commercial use, distribution, and reproduction in any medium, provided the original work is properly cited. 


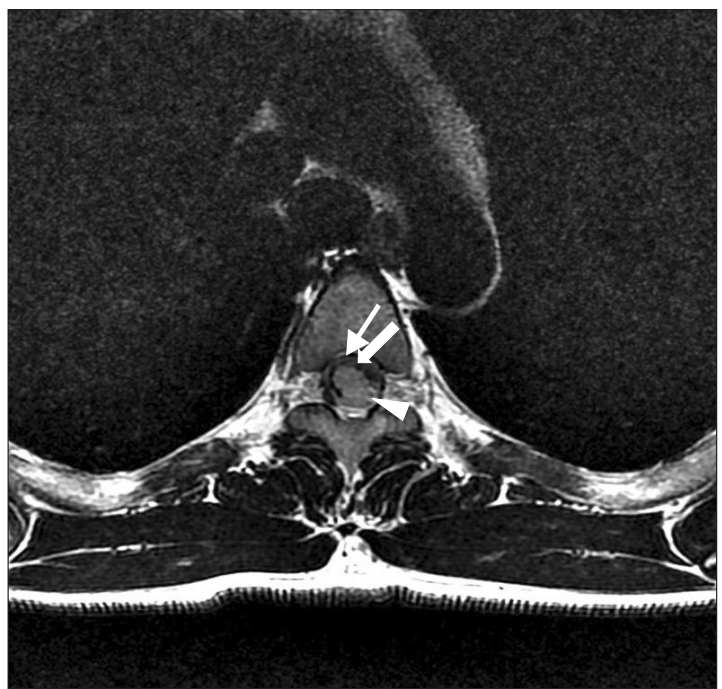

Fig. 1. T2-weighted images on cross section of the upper thoracic intramedullary tumor (T4 level). The mass is located at the posteroleft lateral side of spinal cord (arrow head), surrounded by hypoechoic halo signifying clotted blood, macrophages and glial cells and suggesting cavernous hemangioma (thick arrow). Normal cord tissue compressed by tumor tissue is shown as a thin layer (thin arrow).

effective. We also considered the possibility of neurotoxicity following the injection of heavy bupivacaine, but that idea was discarded because neurotoxicity is presumed to appear bilaterally.

On reconsideration, when extensive abnormal neurologic signs, not localized to one or two nerve roots, occurred, a spinal cord problem should have been considered. However, the fact that the patient did not show any motor functional deficits and the thought of having an observation period of the patient's illness or responsiveness to drug treatments delayed the diagnosis of a myelopathy. We also consider why symptoms were localized to the right side and motor function was intact. We postulate that the mass was located on the postero-left lateral side of the spinal cord and the mass compressed only the ascending sensory pathway, not the anteriorly located descending motor pathway.

There have been few studies regarding the mechanism of myelopathy symptoms, such as paraplegia or paresthesia related to an intramedullary or extramedullary mass after spinal anesthesia [1,2]. Hollis et al. [1] evaluated the results of dural puncture in 50 patients who showed a complete block pattern on the myelogram for the evaluation of various spinal masses, and reported that the incidence of compressive complications following lumbar puncture below a spinal mass was at least $14 \%$ (7 of 50 patients). He postulated the main mechanism as follows: With the potentially obstructing mass, there exists a pressure gradient between upper and lower CSF space causing downward force after the dural puncture below the mass. There are a variety of causes of myelopathy related to lumbar puncture for myelography or spinal anesthesia, including metastatic cancer or tuberculosis of the vertebral body, disrupted herniated intervertebral disk, epidural hematoma, epidural abscess, and intramedullary tumor [3,4]. During their natural course, those variable extramedullary or intramedullary masses were associated with not only motor symptoms such as paraparesis but also sensory deficits of extremities. Furthermore, back pain may appear as the first symptom. Therefore, practitioners should pay close attention to clues regarding history and physical examination before central neuraxial blockade.

This patient did not show any signs related to cavernous hemangioma preoperatively. Therefore, it was difficult to predict the neurological complications. However, some patients with cavernous hemangiomas may show suspicious signs in the natural clinical courses. Therefore, we suggest that a careful preoperative history and neurologic examination are essential. If there are any suspicious findings, MR imaging of the spine should be considered for further evaluation [5], and when a spinal mass is confirmed, neuraxial blockade should be absolutely avoided and substituted with general anesthesia.

\section{References}

1. Hollis PH, Malis LI, Zappulla RA. Neurological deterioration after lumbar puncture below complete spinal subarachnoid block. J Neurosurg 1986; 64: 253-6.

2. Jooma R, Hayward RD. Upward spinal coning: impaction of occult spinal tumours following relief of hydrocephalus. J Neurol Neurosurg Psychiatry 1984; 47: 386-90.

3. Karaaslan P, Candan S, Basaran C. Paraplegia after spinal anesthesia as a result of previously undiagnosed vertebral tuberculosis. Anesth Analg 2006; 102: 1900-1.

4. Kim SH, Song GS, Son DW, Lee SW. Neurologic complications following spinal epidural anesthesia in a patient with spinal intradural extramedullary tumor. J Korean Neurosurg Soc 2010; 46: 544-6.

5. Hogan QH, Hendrix LH. Evaluation of neurologic injury following regional anesthesia. In: Complications of Regional Anesthesia. 2nd ed. Edited by Finucane BT: New York, Springer. 2007, pp 386-409. 